\title{
On optimal precoding in linear vector Gaussian channels with arbitrary input distribution
}

\author{
Miquel Payaró \\ Department of Radiocommunications \\ Centre Tecnològic de Telecomunicacions de Catalunya \\ Castelldefels, Barcelona, Spain \\ Email: miquel.payaro@cttc.cat
}

\author{
Daniel P. Palomar \\ Department of Electronic and Computer Engineering \\ Hong Kong University of Science and Technology \\ Clear Water Bay, Kowloon, Hong Kong \\ Email: palomar@ust.hk
}

\begin{abstract}
The design of the precoder the maximizes the mutual information in linear vector Gaussian channels with an arbitrary input distribution is studied. Precisely, the precoder optimal left singular vectors and singular values are derived. The characterization of the right singular vectors is left, in general, as an open problem whose computational complexity is then studied in three cases: Gaussian signaling, low SNR, and high SNR. For the Gaussian signaling case and the low SNR regime, the dependence of the mutual information on the right singular vectors vanishes, making the optimal precoder design problem easy to solve. In the high SNR regime, however, the dependence on the right singular vectors cannot be avoided and we show the difficulty of computing the optimal precoder through an NPhardness analysis.
\end{abstract}

\section{INTRODUCTION}

In linear vector Gaussian channels with an average power constraint, capacity is achieved by zero-mean Gaussian inputs, whose covariance is aligned with the channel eigenmodes and where the power is distributed among the covariance eigenvalues according to the waterfilling policy [1], [2]. Despite the information theoretic optimality of Gaussian inputs, they are seldom used in practice due to their implementation complexity. Rather, system designers often resort to simple discrete constellations, such as BPSK or QAM.

In this context, the scalar relationship between mutual information and minimum mean square error (MMSE) for linear vector Gaussian channels put forth recently in [3], and extended to the vector case in [4], has become a fundamental tool in transmitter design beyond the Gaussian signaling case.

In [5], the authors derived the optimum diagonal precoder, or power allocation, in quasi-closed form, coining the term mercury/waterfilling. Their results were found for the particular case of a diagonal channel corrupted with AWGN and imposing independence on the components of the input vector. The mercury/waterfilling policy was later extended to nondiagonal channels in [6] through a numerical algorithm.

The linear transmitter design (or linear precoding) problem was recently studied in [7], [8] with a wider scope by considering full (non-diagonal) precoder and channel matrices and arbitrary inputs with possibly dependent components. In [7], [8] the authors gave necessary conditions for the optimal

This work was supported by the RGC 618008 and the TEC2008-06327C03-03/TEC research grants. precoder and optimal transmit covariance matrix and proposed numerical iterative methods to compute a (in general suboptimal) solution. Despite all these research efforts, a general solution for this problem is still missing. In this work, we make a step towards the characterization of its solution and give some hints and ideas on why this problem is so challenging.

The contributions of the present paper are:

1) The expression for the optimal left singular vector matrix of the precoder that maximizes a wide family of objective functions (including the mutual information) is given.

2) We give a necessary and sufficient condition for the optimal singular values of the precoder that maximizes the mutual information and propose an efficient method to numerically compute it.

3) We show that the dependence of the mutual information on the right singular vector matrix of the precoder is a key element in the intractability of computing the precoder that maximizes the mutual information.

4) We give an expression for the Jacobian of the mutual information with respect to the transmitted signal covariance, correcting the expression in [4, Eq. (24)].

Formalism: In this work we define a program according to

$$
\begin{aligned}
& \left\{f_{0}^{\star}, x_{1}^{\star}, \ldots, x_{m}^{\star}\right\}=\operatorname{Name}\left(a_{1}, \ldots, a_{p}\right) \\
& \quad:=\max _{x_{1}, \ldots, x_{m}} f_{0}\left(x_{1}, \ldots, x_{m}, a_{1}, \ldots, a_{p}\right) \\
& \quad \text { subject to } f_{i}\left(x_{1}, \ldots, x_{m}, a_{1}, \ldots, a_{p}\right) \leq 0, \quad \forall i,
\end{aligned}
$$

where $\left(a_{1}, \ldots, a_{p}\right)$ are the parameters and $\left(x_{1}, \ldots, x_{m}\right)$ are the optimization variables. Observe that the first returned argument, $f_{0}^{\star}$, corresponds to the optimal value of the objective function. We also make use of the Jacobian operator D applied to a matrix valued function $\mathbf{F}$ of a matrix argument $\mathbf{X}$ defined as $D_{\mathbf{X}} \mathbf{F}=(\partial v e c \mathbf{F}) /\left(\partial v_{e c}^{\top} \mathbf{X}\right)[9$, Sec. 9.4], where vec $\mathbf{X}$ is the vector obtained stacking the columns of $\mathbf{X}$. This notation requires some modifications when either $\mathbf{F}$ or $\mathbf{X}$ are symmetric matrices, see [9] for details. In Section VI we use some concepts of computational complexity and program reductions. See [10], [11] for reference. 


\section{SigNAL MODEL}

We consider a general discrete-time linear vector Gaussian channel, whose output $\boldsymbol{Y} \in \mathbb{R}^{n}$ is represented by the following signal model

$$
\boldsymbol{Y}=\mathbf{H P} \boldsymbol{S}+\boldsymbol{Z},
$$

where $\boldsymbol{S} \in \mathbb{R}^{m}$ is the input vector distributed according to $P_{\boldsymbol{S}}(\boldsymbol{s})$, the matrices $\mathbf{H} \in \mathbb{R}^{n \times p}$ and $\mathbf{P} \in \mathbb{R}^{p \times m}$ represent the channel and precoder linear transformations, respectively, and $Z \in \mathbb{R}^{n}$ represents a zero-mean Gaussian noise with identity covariance matrix $\mathbf{R}_{Z}=\mathbf{I}^{1}$.

For the sake of simplicity, we assume that $E\{\boldsymbol{S}\}=\mathbf{0}$ and $\mathrm{E}\left\{\boldsymbol{S} \boldsymbol{S}^{\top}\right\}=\mathbf{I}$. The transmitted power $\rho$ is thus given by $\rho=\operatorname{Tr}\left(\mathbf{P P}^{\top}\right)$. We will also make use of the notation $\mathbf{P}=\sqrt{\rho} \overline{\mathbf{P}}$, with $\operatorname{Tr}\left(\overline{\mathbf{P}} \overline{\mathbf{P}}^{\top}\right)=1$ and also define $\mathbf{R}_{\mathbf{H}}=\mathbf{H}^{\top} \mathbf{H}$. Moreover, we define the SVD decomposition of the precoder as $\mathbf{P}=\mathbf{U}_{\mathbf{P}} \boldsymbol{\Sigma}_{\mathbf{P}} \mathbf{V}_{\mathbf{P}}^{\top}$, the entries of $\boldsymbol{\Sigma}_{\mathbf{P}}$ as $\sigma_{i}=\left[\boldsymbol{\Sigma}_{\mathbf{P}}\right]_{i i}$, and also the eigendecomposition of the channel covariance as $\mathbf{R}_{\mathbf{H}}=\mathbf{U}_{\mathbf{H}} \boldsymbol{\Lambda}_{\mathbf{H}}^{2} \mathbf{U}_{\mathbf{H}}^{\top}$. Finally, we define the MMSE matrix as $\mathbf{E}_{\boldsymbol{S}}=\mathrm{E}\left\{(\boldsymbol{S}-\mathrm{E}\{\boldsymbol{S} \mid \boldsymbol{Y}\})(\boldsymbol{S}-\mathrm{E}\{\boldsymbol{S} \mid \boldsymbol{Y}\})^{\top}\right\}$.

\section{Problem Definition And Structure of the SOLUTION}

In this paper we are interested in studying the properties of the precoder $\mathbf{P}$ that maximizes the mutual information under an average transmitted power constraint. However, in this section we consider the more generic problem setup

$$
\begin{aligned}
\left\{\mathcal{P}_{0}^{\star}, \mathbf{P}_{\mathcal{P}_{0}}^{\star}\right\}=\text { MaxPerformace } & \left(\rho, P_{\boldsymbol{S}}(\boldsymbol{s}), \mathbf{R}_{\mathbf{H}}\right) \\
:=\max _{\mathbf{P}} & \mathcal{P}_{0} \\
\text { s.t. } & \operatorname{Tr}\left(\mathbf{P P}^{\top}\right)=\rho,
\end{aligned}
$$

where $\mathcal{P}_{0}$ is a generic performance measure that depends on the precoder $\mathbf{P}$ through the received vector $\boldsymbol{Y}$.

In the following lemma we characterize the dependence of $\mathcal{P}_{0}$ on the precoder matrix $\mathbf{P}$.

Lemma 1: Consider a performance measure $\mathcal{P}_{0}$ of the system $\boldsymbol{Y}=\mathbf{H P} \boldsymbol{S}+\boldsymbol{Z}$, such that $\mathcal{P}_{0}$ depends on the distribution of the random observation $\boldsymbol{Y}$ conditioned on the input $\boldsymbol{S}$. It then follows that the dependence of $\mathcal{P}_{0}$ on the precoder $\mathbf{P}$ is only through $\mathbf{P}^{\top} \mathbf{R}_{\mathbf{H}} \mathbf{P}$ and we can thus write without loss of generality $\mathcal{P}_{0}=\mathcal{P}_{0}\left(\mathbf{P}^{\top} \mathbf{R}_{\mathbf{H}} \mathbf{P}\right)$.

Proof: The proof follows quite easily by noting that $\mathbf{P}^{\top} \mathbf{H}^{\top} \boldsymbol{Y}$ is a sufficient statistic of $\boldsymbol{Y}$, [2, Section 2.10]. The sufficient statistic is thus $\mathbf{P}^{\top} \mathbf{H}^{\top} \mathbf{H P} \boldsymbol{S}+\mathbf{P}^{\top} \mathbf{H}^{\top} \boldsymbol{Z}$. The first term obviously depends on $\mathbf{P}$ only through $\mathbf{P}^{\top} \mathbf{R}_{\mathbf{H}} \mathbf{P}$. Since the second term $\mathbf{P}^{\top} \mathbf{H}^{\top} \boldsymbol{Z}$ is a Gaussian random vector, its behavior is completely determined by its mean (assumed zero) and its covariance matrix, given by $\mathbf{P}^{\top} \mathbf{R}_{\mathbf{H}} \mathbf{P}$.

From all the possible choices for the performance measure function $\mathcal{P}_{0}$, we are now going to focus our attention on the specific class of reasonable performance measures, which is defined next.

\footnotetext{
${ }^{1}$ The assumption $\mathbf{R}_{Z}=\mathbf{I}$ is made w.l.o.g., as, for the case $\mathbf{R}_{Z} \neq \mathbf{I}$, we could always consider the whitened received signal $\mathbf{R}_{Z}^{-1 / 2} \boldsymbol{Y}$.
}

Definition 1: A performance measure $\mathcal{P}_{0}\left(\mathbf{P}^{\top} \mathbf{R}_{\mathbf{H}} \mathbf{P}\right)$ is said to be reasonable if it fulfills that $\mathcal{P}_{0}\left(\alpha \mathbf{P}^{\top} \mathbf{R}_{\mathbf{H}} \mathbf{P}\right)>$ $\mathcal{P}_{0}\left(\mathbf{P}^{\top} \mathbf{R}_{\mathbf{H}} \mathbf{P}\right)$, for any $\alpha>1$ and $\mathbf{P}^{\top} \mathbf{R}_{\mathbf{H}} \mathbf{P} \neq \mathbf{0}$, which implies that $\mathcal{P}_{0}$ is a power efficient performance measure.

Remark 1: The generic cost function ${ }^{2} f_{0}$ considered in [12] was assumed to be a function of the elements of the vector $\operatorname{diag}\left(\left(\mathbf{I}+\mathbf{P}^{\top} \mathbf{R}_{\mathbf{H}} \mathbf{P}\right)^{-1}\right)$ and increasing in each argument. Recalling that, for any $\alpha>1$ and $\mathbf{P}^{\top} \mathbf{R}_{\mathbf{H}} \mathbf{P} \neq \mathbf{0}$, we have

$$
\left[\operatorname{diag}\left(\left(\mathbf{I}+\alpha \mathbf{P}^{\top} \mathbf{R}_{\mathbf{H}} \mathbf{P}\right)^{-1}\right)\right]_{i}<\left[\operatorname{diag}\left(\left(\mathbf{I}+\mathbf{P}^{\top} \mathbf{R}_{\mathbf{H}} \mathbf{P}\right)^{-1}\right)\right]_{i} .
$$

It is straightforward to see that the performance measure defined as $\mathcal{P}_{0} \triangleq-f_{0}$ is a reasonable performance measure according to Definition 1.

Based on a result in [12] for the design of optimal linear precoders, we characterize the left singular vectors of an optimal precoder of (3).

Proposition 1: Consider the optimization problem in (3). It then follows that, for any reasonable performance measure $\mathcal{P}_{0}$, the left singular vectors of the optimal precoder $\mathbf{P} \in \mathbb{R}^{p \times m}$ can always be chosen to coincide with the eigenvectors of the channel covariance $\mathbf{R}_{\mathbf{H}}$ associated with the $\min \{p, m\}$ largest eigenvalues.

Proof: For simplicity we consider the case $m \geq p$. The case $m<p$ follows similarly. From the SVD of the precoder $\mathbf{P}=\mathbf{U}_{\mathbf{P}} \boldsymbol{\Sigma}_{\mathbf{P}} \mathbf{V}_{\mathbf{P}}^{\top}$ and the eigen-decomposition of the matrix

$$
\boldsymbol{\Sigma}_{\mathbf{P}} \mathbf{U}_{\mathbf{P}}^{\top} \mathbf{R}_{\mathbf{H}} \mathbf{U}_{\mathbf{P}} \boldsymbol{\Sigma}_{\mathbf{P}}=\mathbf{Q} \boldsymbol{\Delta} \mathbf{Q}^{\top},
$$

with $\Delta$ diagonal and $\mathbf{Q}$ orthonormal, it follows that

$$
\mathbf{Q}^{\top} \boldsymbol{\Sigma}_{\mathbf{P}} \mathbf{U}_{\mathbf{P}}^{\top} \mathbf{R}_{\mathbf{H}} \mathbf{U}_{\mathbf{P}} \boldsymbol{\Sigma}_{\mathbf{P}} \mathbf{Q}
$$

is a diagonal matrix. From [12, Lemma 12], we can state that there exists a matrix $\mathbf{M}=\mathbf{U}_{\mathbf{H}} \boldsymbol{\Sigma}_{\mathbf{M}}$, with $\boldsymbol{\Sigma}_{\mathbf{M}}$ having non-zero elements only in the main diagonal, such that $\mathbf{M}^{\top} \mathbf{R}_{\mathbf{H}} \mathbf{M}=\boldsymbol{\Delta}$ and that $\operatorname{Tr}\left(\mathbf{M} \mathbf{M}^{\top}\right) \leq \operatorname{Tr}\left(\boldsymbol{\Sigma}_{\mathbf{P}}\right)=\operatorname{Tr}\left(\mathbf{P P}^{\top}\right)$. Now, we only need to check that

$$
\mathbf{P}^{\top} \mathbf{R}_{\mathbf{H}} \mathbf{P}=\mathbf{V}_{\mathbf{P}} \mathbf{Q} \boldsymbol{\Delta} \mathbf{Q}^{\top} \mathbf{V}_{\mathbf{P}}^{\top}=\mathbf{V}_{\mathbf{P}} \mathbf{Q} \mathbf{M}^{\top} \mathbf{R}_{\mathbf{H}} \mathbf{M} \mathbf{Q}^{\top} \mathbf{V}_{\mathbf{P}}^{\top}
$$

Defining $\widetilde{\mathbf{P}}=\mathbf{M Q}^{\top} \mathbf{V}_{\mathbf{P}}^{\top}=\mathbf{U}_{\mathbf{H}} \boldsymbol{\Sigma}_{\mathbf{M}} \widetilde{\mathbf{V}}^{\top}$, with $\widetilde{\mathbf{V}}=\mathbf{V}_{\mathbf{P}} \mathbf{Q}$, we have shown by construction that for any given matrix $\mathbf{P}$ we can find another matrix $\widetilde{\mathbf{P}}$ such that the objective function in (3) is the same,

$$
\widetilde{\mathbf{P}}^{\top} \mathbf{R}_{\mathbf{H}} \widetilde{\mathbf{P}}=\mathbf{P}^{\top} \mathbf{R}_{\mathbf{H}} \mathbf{P} \Rightarrow \mathcal{P}_{0}\left(\widetilde{\mathbf{P}}^{\top} \mathbf{R}_{\mathbf{H}} \widetilde{\mathbf{P}}\right)=\mathcal{P}_{0}\left(\mathbf{P}^{\top} \mathbf{R}_{\mathbf{H}} \mathbf{P}\right),
$$

which follows from Lemma 1, whereas the required transmitted power is not larger, $\operatorname{Tr}\left(\widetilde{\mathbf{P}} \widetilde{\mathbf{P}}^{\top}\right)=\operatorname{Tr}\left(\mathbf{M} \mathbf{M}^{\top}\right) \leq \operatorname{Tr}\left(\mathbf{P} \mathbf{P}^{\top}\right)$. Since the performance measure $\mathcal{P}_{0}$ is reasonable, the result follows directly.

From the result in Proposition 1, it follows that, the channel model in (2) can be simplified, without loss of optimality, to

$$
\boldsymbol{Y}^{\prime}=\boldsymbol{\Lambda}_{\mathbf{H}} \boldsymbol{\Sigma}_{\mathbf{P}} \mathbf{V}_{\mathbf{P}}^{\top} \boldsymbol{S}+\boldsymbol{Z}
$$

where now the only optimization variables are $\boldsymbol{\Sigma}_{\mathbf{P}}$ and $\mathbf{V}_{\mathbf{P}}$.

\footnotetext{
${ }^{2}$ Observe that, while a performance measure $\mathcal{P}_{0}$ is to be maximized, a cost function $f_{0}$ is usually to be minimized.
} 


\section{OPTIMAL SINGULAR VALUES}

In this section we particularize the generic performance measure considered in the previous section to the input-output mutual information in $(8)$, i.e., $\mathcal{P}_{0}=I\left(\boldsymbol{S} ; \boldsymbol{Y}^{\prime}\right)$. To compute the optimal $\boldsymbol{\Sigma}_{\mathbf{P}}^{\star}$ we define

$$
\begin{gathered}
\left\{I^{\star}, \boldsymbol{\Sigma}_{\mathbf{P}}^{2 \star}\right\}=\text { OptPowerAlloc }\left(\rho, P_{\boldsymbol{S}}(\boldsymbol{s}), \boldsymbol{\Lambda}_{\mathbf{H}}, \mathbf{V}_{\mathbf{P}}\right) \\
:=\max _{\left\{\sigma_{i}^{2}\right\}} I\left(\boldsymbol{S} ; \boldsymbol{Y}^{\prime}\right) \\
\text { s.t. } \sum_{i} \sigma_{i}^{2}=\rho .
\end{gathered}
$$

Observe that the optimization is done with respect to the optimal squared singular values. The optimal singular values are then defined up to a sign, which does not affect the mutual information. Consequently, we define $\sigma_{i}^{\star}=+\sqrt{\sigma_{i}^{2 \star}}$ and $\left[\boldsymbol{\Sigma}_{\mathbf{P}}^{\star}\right]_{i i}=\sigma_{i}^{\star}$.

Let us now present an appealing property of $I\left(\boldsymbol{S} ; \boldsymbol{Y}^{\prime}\right)$.

Lemma 2 ([13]): Consider the model in (8) and fix $\mathbf{V}_{\mathbf{P}}$. Then it follows that the mutual information $I\left(\boldsymbol{S} ; \boldsymbol{Y}^{\prime}\right)$ is a concave function of the squared diagonal entries of $\boldsymbol{\Sigma}_{\mathbf{P}}$.

With this result, we can now obtain a necessary and sufficient condition for the squared entries of $\boldsymbol{\Sigma}_{\mathbf{P}}^{\star}$.

Proposition 2: The entries of the squared singular value matrix $\sigma_{i}^{2 \star}=\left[\boldsymbol{\Sigma}_{\mathbf{P}}^{2 \star}\right]_{i i}$ of the solution to (9) satisfy

$$
\begin{aligned}
\sigma_{i}^{2 \star}=0 & \Rightarrow \quad\left[\boldsymbol{\Lambda}_{\mathbf{H}}^{2}\right]_{i i} \mathrm{mmse}_{i}\left(\boldsymbol{\Sigma}_{\mathbf{P}}^{\star}, \mathbf{V}_{\mathbf{P}}\right)<2 \eta \\
\sigma_{i}^{2 \star}>0 & \Rightarrow \quad\left[\boldsymbol{\Lambda}_{\mathbf{H}}^{2}\right]_{i i} \mathrm{mmse}_{i}\left(\boldsymbol{\Sigma}_{\mathbf{P}}^{\star}, \mathbf{V}_{\mathbf{P}}\right)=2 \eta,
\end{aligned}
$$

where $\eta$ is such that the power constraint is satisfied and where we have used $\operatorname{mmse}_{i}\left(\boldsymbol{\Sigma}_{\mathbf{P}}^{\star}, \mathbf{V}_{\mathbf{P}}\right)$ to define the $i$-th diagonal entry of the MMSE matrix $\mathbf{E}_{\widehat{S}}$ corresponding to the model $\boldsymbol{Y}^{\prime}=\Lambda_{\mathbf{H}} \boldsymbol{\Sigma}_{\mathrm{P}}^{\star} \widehat{\boldsymbol{S}}+\boldsymbol{Z}$ with $\widehat{\boldsymbol{S}}=\mathbf{V}_{\mathbf{P}}^{\top} \boldsymbol{S}$.

Proof: The proof is based on obtaining the KKT conditions of the optimization problem in (9) together with

$$
\begin{aligned}
& \frac{\mathrm{d} I\left(\boldsymbol{S} ; \boldsymbol{\Lambda}_{\mathbf{H}} \boldsymbol{\Sigma}_{\mathbf{P}} \widehat{\boldsymbol{S}}+\boldsymbol{Z}\right)}{\mathrm{d}\left(\sigma_{i}^{2}\right)} \\
& =\left[\boldsymbol{\Lambda}_{\mathbf{H}}^{2}\right]_{i i} \mathrm{E}\left\{\left([\widehat{\boldsymbol{S}}]_{i}-\mathrm{E}\left\{[\widehat{\boldsymbol{S}}]_{i} \mid \boldsymbol{\Lambda}_{\mathbf{H}} \boldsymbol{\Sigma}_{\mathbf{P}}^{\star} \widehat{\boldsymbol{S}}+\boldsymbol{Z}\right\}\right)^{2}\right\},
\end{aligned}
$$

which follows from [4, Cor. 2].

Remark 2: The set of non-linear equations in (10) can be numerically solved with, e.g., the Newton method because it has quadratic convergence and the concavity property stated in Lemma 2 guarantees the global optimality of the obtained solution. The expression for the entries of the Jacobian vector of mmse $_{i}\left(\boldsymbol{\Sigma}_{\mathbf{P}}^{\star}, \mathbf{V}_{\mathbf{P}}\right)$ with respect to the squared entries of $\boldsymbol{\Sigma}_{\mathbf{P}}$, which is needed at each iteration, is given by [13]

$$
\frac{\mathrm{dmmse}_{i}\left(\boldsymbol{\Sigma}_{\mathbf{P}}^{\star}, \mathbf{V}_{\mathbf{P}}\right)}{\mathrm{d}\left(\sigma_{j}^{2}\right)}=-\left[\boldsymbol{\Lambda}_{\mathbf{H}}^{2}\right]_{j j} \mathrm{E}\left\{\left[\boldsymbol{\Phi}\left(\boldsymbol{Y}^{\prime}\right)\right]_{i j}^{2}\right\},
$$

where $\boldsymbol{\Phi}\left(\boldsymbol{y}^{\prime}\right)=\mathrm{E}\left\{\widehat{\boldsymbol{S}} \widehat{\boldsymbol{S}}^{\top} \mid \boldsymbol{y}^{\prime}\right\}-\mathrm{E}\left\{\widehat{\boldsymbol{S}} \mid \boldsymbol{y}^{\prime}\right\} \mathrm{E}\left\{\widehat{\boldsymbol{S}}^{\top} \mid \boldsymbol{y}^{\prime}\right\}$.

At this point, we have obtained the optimal left singular vectors and the optimal singular values of the linear precoder that maximizes the mutual information for a fixed $\mathbf{V}_{\mathbf{P}}$. Unfortunately, the optimal solution for the right singular vectors $\mathbf{V}_{\mathbf{P}}$ seems to be an extremely difficult problem. A simple suboptimal solution consists in optimizing $\mathbf{V}_{\mathbf{P}}$ based on standard numerical methods guaranteed to converge to a local optimum. See further [14] for details on the practical algorithm to compute the precoder.

From the results presented in this section, it is apparent that the difficulty of the problem in (3) when optimizing the mutual information lies in the computation of the optimal right singular vectors matrix, $\mathbf{V}_{\mathbf{P}}^{\star}$. To support this statement, in the following sections we deal with three cases: the Gaussian signaling case, and the low and high SNR regimes. In the Gaussian signaling case and low SNR regime, we recover the well-known result that the mutual information depends only on the squared precoder $\mathbf{Q}_{\mathbf{P}}=\mathbf{P P}^{\top}$ and is independent of the right singular vectors matrix $\mathbf{V}_{\mathbf{P}}$, which further implies that, in both cases, the optimal precoder can be easily computed. In Section VI we will show that, for the high SNR regime, the precoder design problem becomes computationally difficult through a NP-hardness analysis.

\section{SitUATIONS WHERE THE MUTUAL INFORMATION IS INDEPENDENT OF $\mathbf{V}_{\mathbf{P}}$}

1) Gaussian signaling case: For the Gaussian signaling case, we recover the well known expression for the mutual information [2]

$$
I(\boldsymbol{S} ; \boldsymbol{Y})=\frac{1}{2} \log \operatorname{det}\left(\mathbf{I}+\mathbf{Q}_{\mathbf{P}} \mathbf{R}_{\mathbf{H}}\right),
$$

from which it is clear that the only dependence of the mutual information on the precoder is through $\mathbf{Q}_{\mathbf{P}}=\mathbf{U}_{\mathbf{P}} \boldsymbol{\Sigma}_{\mathbf{P}}^{2} \mathbf{U}_{\mathbf{P}}^{\top}$ and, thus, it is independent of $\mathbf{V}_{\mathbf{P}}$. As we have pointed out in the introduction and generalized in Proposition 1, the optimal covariance $\mathbf{Q}_{\mathbf{P}}$ is aligned with the channel eigenmodes $\mathbf{U}_{\mathbf{H}}$. Also the power is distributed among the covariance eigenvalues $\boldsymbol{\Sigma}_{\mathbf{P}}^{2}$ according to the waterfilling policy [1], which can be computed efficiently.

2) Low SNR regime: For the low SNR regime, a first-order expression of the mutual information is [4]

$$
I(\boldsymbol{S} ; \boldsymbol{Y})=\frac{1}{2} \operatorname{Tr}\left(\mathbf{Q}_{\mathbf{P}} \mathbf{R}_{\mathbf{H}}\right)+o\left(\left\|\mathbf{Q}_{\mathbf{P}}\right\|\right) .
$$

Just as in the previous case, from this expression it is clear that the mutual information is insensitive to the right singular vector matrix $\mathbf{V}_{\mathbf{P}}$. Moreover, the optimal matrix $\mathbf{Q}_{\mathbf{P}}$ is easy to obtain in closed form $[15]^{3}$.

Remark 3: The expression in (13) was derived in [4] through the expression of the Jacobian of the mutual information with respect to $Q_{\mathbf{P}}$. Although the result in (13) is correct, the expression for the Jacobian $\mathrm{D}_{\mathrm{Q}_{\mathbf{P}}} I(\boldsymbol{S} ; \boldsymbol{Y})$ given in [4, Eq. (24)] is only valid in the low SNR regime. The correct expression for $\mathrm{D}_{\mathrm{Q}_{\mathrm{P}}} I(\boldsymbol{S} ; \boldsymbol{Y})$ valid for all SNRs is [16]

$$
\begin{aligned}
& \mathrm{D}_{\mathbf{Q}_{\mathbf{P}}} I(\boldsymbol{S} ; \boldsymbol{Y})=\frac{1}{2} \operatorname{vec}^{\top}\left(\mathbf{R}_{\mathbf{H}} \mathbf{P} \mathbf{E}_{\boldsymbol{S}} \mathbf{P}^{-1}\right) \mathbf{D}_{n} \\
& \quad-\operatorname{vec}^{\top}\left(\mathbf{E}_{\boldsymbol{S}} \mathbf{P}^{\top} \mathbf{R}_{\mathbf{H}} \mathbf{U}_{\mathbf{P}} \boldsymbol{\Sigma}_{\mathbf{P}}\right) \boldsymbol{\Omega}_{n}\left(\mathbf{P}^{-1} \otimes \mathbf{P}^{\top}\right) \mathbf{D}_{n},
\end{aligned}
$$

\footnotetext{
${ }^{3}$ The optimal signaling strategy in the low SNR regime was studied in full generality in [15]. We recall that, in this work, we are assuming that the signaling is fixed and the only remaining degree of freedom to maximize the mutual information is the precoder matrix $\mathbf{P}$.
} 
with

$$
\boldsymbol{\Omega}=\left(\begin{array}{c}
\mathbf{v}_{1}^{\top} \otimes \mathbf{V}_{\mathbf{P}}\left(\sigma_{1}^{2} \mathbf{I}-\boldsymbol{\Sigma}_{\mathbf{P}}^{2}\right)^{+} \mathbf{V}_{\mathbf{P}}^{\top} \\
\mathbf{v}_{2}^{\top} \otimes \mathbf{V}_{\mathbf{P}}\left(\sigma_{2}^{2} \mathbf{I}-\boldsymbol{\Sigma}_{\mathbf{P}}^{2}\right)^{+} \mathbf{V}_{\mathbf{P}}^{\top} \\
\vdots \\
\mathbf{v}_{n}^{\top} \otimes \mathbf{V}_{\mathbf{P}}\left(\sigma_{n}^{2} \mathbf{I}-\boldsymbol{\Sigma}_{\mathbf{P}}^{2}\right)^{+} \mathbf{V}_{\mathbf{P}}^{\top}
\end{array}\right)
$$

where $\mathbf{v}_{i}$ is the $i$-th column of matrix $\mathbf{V}_{\mathbf{P}}, \mathbf{N}_{n}$ and $\mathbf{D}_{n}$ are the symmetrization and duplication matrices defined in $[9$, Secs. 3.7, 3.8], $\mathbf{A}^{+}$denotes the Moore-Penrose pseudo-inverse, and where for the sake of clarity, we have assumed that $\mathbf{P}^{-1}$ exists and that $n=m=p$.

\section{HIGH SNR REGIME}

In this section we consider that the signaling is discrete, i.e., the input can only take values from a finite set, $\boldsymbol{S} \in \mathcal{S} \triangleq$ $\left\{\mathbf{s}^{(i)}\right\}_{i=1}^{L}$. As discussed in [5], [8], for discrete inputs and high SNR, the maximization of the problem in (3) with the mutual information as performance measure is asymptotically equivalent to the maximization of the squared minimum distance, $d_{\min }{ }^{4}$, among the received constellation points defined as $d_{\text {min }}=\min _{\mathbf{e} \in \mathcal{E}} \mathbf{e}^{\top} \mathbf{P}^{\top} \mathbf{R}_{\mathbf{H}} \mathbf{P e}$, where $\mathcal{E}$ is the set containing all the possible differences between the input points in $\mathcal{S}$.

Consequently, let us begin by considering the optimization problem of finding the precoder that maximizes the minimum distance among the received constellation points

$$
\begin{array}{r}
\left\{d^{\star}, \mathbf{P}_{d}^{\star}\right\}=\text { MaxMinDist }(\rho, \mathcal{E}, \mathbf{H}) \\
:=\max _{\mathbf{P}} \min _{\mathbf{e} \in \mathcal{E}} \mathbf{e}^{\top} \mathbf{P}^{\top} \mathbf{R}_{\mathbf{H}} \mathbf{P e} \\
\text { s.t. } \operatorname{Tr}\left(\mathbf{P} \mathbf{P}^{\top}\right)=\rho .
\end{array}
$$

In the following, we give the proof that the program in (16) is NP-hard with respect to the dimension $m$ of the signaling vector, $\boldsymbol{S} \in \mathbb{R}^{m}$, for the case where the set $\mathcal{E}$ is considered to be unstructured (i.e., not constrained to be a difference set). We are now preparing the proof without this assumption in [14]. The proof is based on a series of Cook reductions. We say that program $A$ can be Cook reduced to program $B, A \stackrel{\text { CoOK }}{\longrightarrow} B$, if program $A$ can be computed with a polynomial time algorithm that calls program $B$ as a subroutine assuming that the call is performed in one clock cycle. We have that, if $A \stackrel{\text { COoK }}{\longrightarrow} B$ and A is NP-hard, then B is also in NP-hard, [11].

Before giving the actual proof we describe two more programs and give some of their properties.

\section{A. Intermediate programs and their properties}

We first present the MinNorm program, which computes the minimum norm vector that fulfills a set of constraints on its scalar product with a given set of vectors $\left\{\mathbf{w}_{i}\right\}_{i=1}^{m}$

$$
\begin{aligned}
\left\{t^{\star}, \mathbf{z}^{\star}\right\}=\operatorname{MinNorm} & \left(\left\{\mathbf{w}_{i}\right\}_{i=1}^{m}\right) \\
:=\min _{\mathbf{z} \in \mathbb{R}^{m}}\|\mathbf{z}\|^{2} & \text { s.t. }\left|\mathbf{w}_{i}^{\top} \mathbf{z}\right| \geq 1, \quad i=1, \ldots, m .
\end{aligned}
$$

Lemma 3 ([17]): MinNorm is NP-hard.

\footnotetext{
${ }^{4}$ Although we use the symbol $d_{\min }$, it denotes squared distance.
}

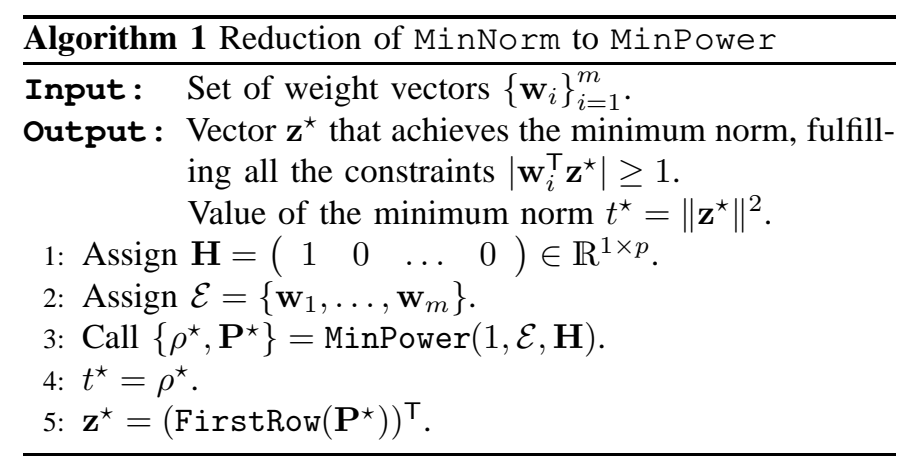

The second problem is MinPower and it computes the precoder that minimizes the transmitted power such that the minimum distance is above a certain threshold:

$$
\begin{aligned}
\left\{\rho^{\star}, \mathbf{P}^{\star}\right\}=\text { MinPower } & (d, \mathcal{E}, \mathbf{H}) \\
& :=\min _{\mathbf{P}} \operatorname{Tr}\left(\mathbf{P} \mathbf{P}^{\top}\right) \\
& \text { s.t. } \min _{\mathbf{e} \in \mathcal{E}} \mathbf{e}^{\top} \mathbf{P}^{\top} \mathbf{R}_{\mathbf{H}} \mathbf{P e} \geq d .
\end{aligned}
$$

Lemma 4: Assume that $\left\{d_{0}^{\star}, \mathbf{P}_{0}^{\star}\right\}$ is the output to the program MaxMinDist $\left(\rho_{0}, \mathcal{E}, \mathbf{H}\right)$. It then follows that the output to MinPower $\left(d_{0}^{\star}, \mathcal{E}, \mathbf{H}\right)$ is given by $\left\{\rho_{0}, \mathbf{P}_{0}^{\star}\right\}$.

Similarly, assume that $\left\{\rho_{0}^{\star}, \mathbf{P}_{0}^{\star}\right\}$ is the output to the program MinPower $\left(d_{0}, \mathcal{E}, \mathbf{H}\right)$. It then follows that the output to MaxMinDist $\left(\rho_{0}^{\star}, \mathcal{E}, \mathbf{H}\right)$ is given by $\left\{d_{0}, \mathbf{P}_{0}^{\star}\right\}$.

Proof: See [18].

Lemma 5: Assume that $\left\{d_{0}^{\star}, \mathbf{P}_{0}^{\star}\right\}$ is the output to the program MaxMinDist $\left(\rho_{0}, \mathcal{E}, \mathbf{H}\right)$. It then follows that the output to MaxMinDist $\left(\alpha \rho_{0}, \mathcal{E}, \mathbf{H}\right)$ with $\alpha>0$ is given by $\left\{\alpha d_{0}^{\star}, \sqrt{\alpha} \mathbf{P}_{0}^{\star}\right\}$.

Proof: The proof follows easily, e.g., by considering the change of optimization variable $\mathbf{P}=\sqrt{\alpha} \widetilde{\mathbf{P}}$ and noting that the solution to the optimization problem remains unchanged if the objective function is scaled by a constant parameter.

In the following we prove the following chain of reductions: MinNorm $\stackrel{\text { CoOK }}{\longrightarrow}$ MinPower $\stackrel{\text { CoOK }}{\longrightarrow}$ MaxMinDist.

\section{B. Reduction of MinNorm to MinPower}

In Algorithm 1 we present our proposed Cook reduction of MinNorm to MinPower.

Proposition 3: Algorithm 1 is a polynomial time Cook reduction of MinNorm to MinPower.

Proof: Under the assumption that MinPower can be solved in one clock cycle, it follows that Algorithm 1 runs in polynomial time as well. It remains to check that the output of the algorithm corresponds to the solution to MinNorm.

Note that for the particular values assigned to the channel matrix $\mathbf{H}$ and the set $\mathcal{E}$ in Steps 1 and 2 in Algorithm 1, the program MinPower $(1, \mathcal{E}, \mathbf{H})$ in (18) particularizes to

$$
\begin{array}{ll}
\min _{\mathbf{P}} & \operatorname{Tr}\left(\mathbf{P P}^{\boldsymbol{\top}}\right) \\
\text { s.t. } & \min _{i \in[1, m]} \mathbf{w}_{i}^{\boldsymbol{\top}} \mathbf{p}_{1} \mathbf{p}_{1}^{\boldsymbol{\top}} \mathbf{w}_{i} \geq 1,
\end{array}
$$

where $\mathbf{p}_{1}$ is a column vector with the elements of the first row of the precoder matrix $\mathbf{P}$. Observing that the constraint in (20) 


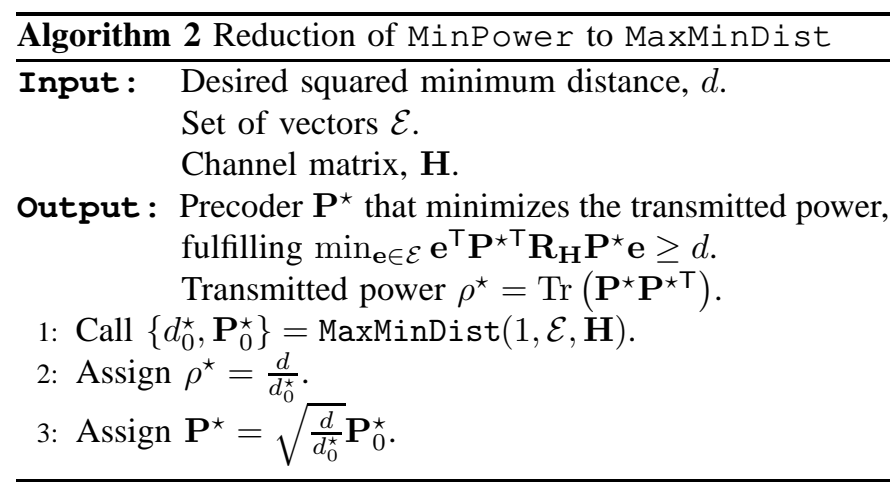

only affects the elements of the first row of matrix $\mathbf{P}$, it is clear that the optimal solution to (19) fulfills $\left[\mathbf{P}^{\star}\right]_{i j}=0, \forall i \neq 1$, as this assignment minimizes the transmitted power. Recalling that $\mathbf{w}_{i}^{\top} \mathbf{p}_{1} \mathbf{p}_{1}^{\top} \mathbf{w}_{i}=\left|\mathbf{w}_{i}^{\top} \mathbf{p}_{1}\right|^{2}$, it is now straightforward to see that the first row of matrix $\mathbf{P}^{\star}$, which is the solution to the problem in (19), is also the solution to MinNorm in (17).

Corollary 1: For the case where the set $\mathcal{E}$ is unconstrained, the program MinPower is NP-hard.

\section{Reduction of MinPower to MaxMinDist}

In Algorithm 2 we present our proposed Cook reduction of MinPower to MaxMinDist.

Proposition 4: Algorithm 2 is a polynomial time Cook reduction of MinPower to MaxMinDist.

Proof: Under the assumption that MaxMinDist can be solved in one clock cycle, it follows that Algorithm 2 runs in polynomial time as well. It remains to check that the output of the algorithm corresponds to the solution to MinPower.

Assume that the output to MaxMinDist $(1, \mathcal{E}, \mathbf{H})$ is given by $\left\{d_{0}^{\star}, \mathbf{P}_{0}^{\star}\right\}$ as in Step 1 in Algorithm 2. Note that, from the power constraint in (16), we have that $\operatorname{Tr}\left(\mathbf{P}_{0}^{\star} \mathbf{P}_{0}^{\star \top}\right)=1$. From Lemma 5, choosing $\alpha=d / d_{0}^{\star}$, it follows that

$$
\left\{d, \sqrt{d / d_{0}^{\star}} \mathbf{P}_{0}^{\star}\right\}=\operatorname{MaxMinDist}\left(d / d_{0}^{\star}, \mathcal{E}, \mathbf{H}\right) \text {. }
$$

Now, applying Lemma 4, we have that

$$
\left\{d / d_{0}^{\star}, \sqrt{d / d_{0}^{\star}} \mathbf{P}_{0}^{\star}\right\}=\operatorname{MinPower}(d, \mathcal{E}, \mathbf{H}),
$$

from which it immediately follows that $\rho^{\star}=d / d_{0}^{\star}$ and $\mathbf{P}^{\star}=$ $\sqrt{d / d_{0}^{\star}} \mathbf{P}_{0}^{\star}$, which completes the proof.

Corollary 2: For the case where the set $\mathcal{E}$ is unconstrained, the program MaxMinDist is NP-hard.

Although the fact that the program MaxMinDist is NP-hard is not a proof that the maximization of the mutual information is also NP-hard, it gives a powerful hint on its expected computational complexity in the high SNR regime where the minimum distance is the key performance parameter.

From this expected complexity on the precoder design at high SNR and the fact that, in Section III, we characterized the optimal left singular vectors and the singular values of the precoder that maximizes the mutual information as a function of the right singular vector matrix $\mathbf{V}_{\mathbf{P}}$, it seems reasonable to place the computational complexity burden of the optimal precoder design in the computation of $\mathbf{V}_{\mathbf{P}}^{\star}$.

\section{CONCLUSION}

We have studied the problem of finding the precoder that maximizes the mutual information for an arbitrary (but given) input distribution. We have found a closed-form expression for the left singular vectors of the optimal precoder and have given a sufficient and necessary condition to compute the optimal singular values. We have also recalled that, in the low SNR or Gaussian signaling scenarios, the optimal precoder can be easily found as the mutual information does not depend on the right singular vectors. Finally, we have argued that in the high SNR regime, the computational complexity of the calculation of the optimal right singular vectors is expected to be hard.

\section{REFERENCES}

[1] E. Telatar, "Capacity of multi-antenna Gaussian channels," European Trans. on Telecomm., vol. 10, no. 6, pp. 585-595, Nov.-Dec. 1999.

[2] T. M. Cover and J. A. Thomas, Elements of Information Theory. New York: John Wiley \& Sons, 1991.

[3] D. Guo, S. Shamai, and S. Verdú, "Mutual information and minimum mean-square error in Gaussian channels," IEEE Trans. on Information Theory, vol. 51, no. 4, pp. 1261-1282, 2005.

[4] D. P. Palomar and S. Verdú, "Gradient of mutual information in linear vector Gaussian channels," IEEE Trans. on Information Theory, vol. 52, no. 1, pp. 141-154, 2006.

[5] A. Lozano, A. Tulino, and S. Verdú, "Optimum power allocation for parallel Gaussian channels with arbitrary input distributions," IEEE Trans. on Information Theory, vol. 52, no. 7, pp. 3033-3051, July 2006.

[6] F. Pérez-Cruz, M. R. D. Rodrigues, and S. Verdú, "Generalized mercury/waterfilling for multiple-input multiple-output channels," in Proc. of the 45th Annual Allerton Conference on Communication, Control, and Computing, Allerton (Illinois, USA), Sept. 2007.

[7] —_, "Optimal precoding for digital subscriber lines," in Proc. IEEE International Conference on Communications (ICC'08), 2008.

[8] M. R. D. Rodrigues, F. Pérez-Cruz, and S. Verdú, "Multipleinput multiple-output Gaussian channels: Optimal covariance for nonGaussian inputs," in Proc. IEEE Information Theory Workshop (ITW '08), 2008, pp. 445-449.

[9] J. Magnus and H. Neudecker, Matrix Differential Calculus with Applications in Statistics and Econometrics, 3rd ed. New York: Wiley, 2007.

[10] C. H. Papadimitriou, Computational Complexity. Reading, Mass: Addison-Wesley, 1994

[11] O. Goldreich, Computational Complexity: A Conceptual Perspective. Cambridge: Cambridge University Press, 2008.

[12] D. P. Palomar, J. M. Cioffi, and M. A. Lagunas, "Joint Tx-Rx beamforming design for multicarrier MIMO channels: A unified framework for convex optimization," IEEE Trans. on Signal Processing, vol. 51, no. 9, pp. 2381-2401, Sep. 2003.

[13] M. Payaró and D. P. Palomar, "Hessian and concavity of mutual information, entropy, and entropy power in linear vector Gaussian channels," accepted in IEEE Trans. on Information Theory, 2009. [Online]. Available: http://arxiv.org/abs/0903.1945

[14] _ "On linear precoding strategies in linear vector Gaussian channels with perfect channel state information," in preparation, 2009.

[15] S. Verdú, "Spectral efficiency in the wideband regime," IEEE Trans. on Information Theory, vol. 48, no. 6, pp. 1319 - 1343, 2002.

[16] M. Payaró and D. P. Palomar, "A note on "Gradient of mutual information in linear vector Gaussian channels"," unpublished document (available upon request), 2008.

[17] Z.-Q. Luo, N. D. Sidiropoulos, P. Tseng, and S. Zhang, "Approximation bounds for quadratic optimization with homogeneous quadratic constraints," SIAM J. on Optimization, vol. 18, no. 1, pp. 1-28, 2007.

[18] S. P. Boyd and L. Vandenberghe, Convex Optimization. Cambridge University Press, 2004. 